\title{
THE HAAR FUNCTIONS ALMOST DIAGONALIZE MULTIPLICATION BY $x$
}

\author{
JOEL ANDERSON ${ }^{1}$
}

\begin{abstract}
It is shown that if $A$ is multiplication by $x$ on $L^{2}(0,1)$, then the matrix for $A$ given by the Haar functions has the form diagonal plus Hilbert-Schmidt.
\end{abstract}

If $A$ is a self-adjoint operator on a complex separable Hilbert space $\mathcal{H}$, then $A$ has the form $D+K$, where $D$ is diagonal (i.e., the eigenvectors for $D$ span $\mathcal{H}$ ) and $K$ is a Hilbert-Schmidt operator. This fact, which was first proved by von Neumann in [1], has played an important role in several areas of analysis. From the proof, however, there is no reason to expect that the basis that "almost diagonalizes" $A$ will have a "nice" form. The purpose of this note is to show there is a natural basis (the Haar functions) for $L^{2}(0,1)$ that almost diagonalizes the operator $A$ defined by

$$
(A f)(x)=x f(x), \quad f \in L^{2}(0,1) .
$$

If $0 \leqslant a \leqslant b \leqslant 1$, write $[a, b]$ for the characteristic function of the indicated interval. The Haar functions are defined as follows:

$$
\varphi_{00}=[0,1], \quad \varphi_{01}=\left[0, \frac{1}{2}\right]-\left[\frac{1}{2}, 1\right]
$$

and, if $n \geqslant 1,0 \leqslant k \leqslant 2^{n}-1$,

$$
\varphi_{n k}=(\sqrt{2})^{n}\left(\left[k / 2^{n}, k / 2^{n}+1 / 2^{n+1}\right]-\left[k / 2^{n}+1 / 2^{n+1},(k+1) / 2^{n}\right]\right) .
$$

It is obvious that the Haar functions form an orthonormal set; and, as their linear span contains the characteristic function of every dyadic subinterval, they form an orthonormal basis.

For each $n$ and $k$ write

$$
a_{n k}=\left\|A \varphi_{n k}\right\|^{2}-\left(A \varphi_{n k}, \varphi_{n k}\right)^{2} .
$$

Easy calculations show $a_{00}=a_{01}=\frac{1}{12}$ and if $n \geqslant 1,0 \leqslant k \leqslant 2^{n}-1$, then $\left\|A \varphi_{n k}\right\|^{2}=\left(\frac{1}{3}\right)\left(2^{-2 n}\right)\left(3 k^{2}+3 k+1\right)$ and $\left(A \varphi_{n k}, \varphi_{n k}\right)=\left(\frac{1}{2}\right)\left(2^{-n}\right)(2 k+1)$.

Received by the editors May 31, 1978.

AMS (MOS) subject classifications (1970). Primary 47A55, 47B15; Secondary 47B10.

Key words and phrases. Haar functions, diagonal plus Hilbert-Schmidt, multiplication by $x$, compact perturbation.

'Research supported in part by a grant from the National Science Foundation. 
Therefore, $a_{n k}=\left(\frac{1}{12}\right)\left(2^{-2 n}\right)$ and

$$
a_{00}+a_{01}+\sum_{n=1}^{\infty} \sum_{k=0}^{2^{n}-1} a_{n k}=\frac{1}{12}+\frac{1}{12}+\sum_{n=1}^{\infty}\left(2^{n}\right)\left(2^{-2 n}\right)\left(\frac{1}{12}\right)=\frac{1}{4} .
$$

If $K$ denotes the operator whose matrix in the basis $\left\{\varphi_{n k}\right\}$ is 0 on the main diagonal and agrees with the matrix for $A$ at the off-diagonal entries, then

$$
\|K\|_{H S}^{2}=\sum a_{n k}=\frac{1}{4} \text {. }
$$

Thus, $K$ is a Hilbert-Schmidt operator and $D=A-K$ is diagonal.

\section{REFERENCES}

1. J. von Neumann, Characterisierung des Spectrums eines Integraloperators, Hermann, Paris, 1935.

Departmeant of Mathematics, Pennsylvania State University, University Park, PennSYLVANIA 16802 